\title{
Geodesics around oscillatons made of exponential scalar field potential
}

\author{
Ali. Mahmoodzadeh* ${ }^{*}$ B. Malakolkalami ${ }^{\dagger}$ \\ Faculty of Science, University of Kurdistan, Sanandaj, P. O. Box :416, Iran, \\ Tel : +988716660066
}

\begin{abstract}
Oscillatons are spherically symmetric solutions to the Einstein-KleinGordon (EKG) equations for soliton stars made of real time-dependent scalar fields. These equations are non singular and satisfy flatness conditions asymptotically with periodic time dependency. In this paper, we investigate the geodesic motion of particles moving around an oscillaton related to a time-dependent scalar field. Bound orbital is found for these particles under the condition of particular values of angular momentum $L$ and initial radial position $r_{0}$. We discuss this topic for an exponential scalar field potential which could be of the form of $V(\Phi)=V_{0} e^{\lambda \sqrt{k_{0}} \Phi}$ with a scalar field $\Phi(r, t)$ and investigate whether the radial coordinates of such particles oscillate in time or not and thereby we could predict the corresponding oscillating period as well as amplitude. It is necessary to recall, in general relativity, a geodesic generalizes the notion of a "straight line" to curved space-time. Importantly, the world line of a particle free from all external, non-gravitational forces, is a particular type of geodesic. In other words, a freely moving or falling particle always moves along a geodesic. In general relativity, gravity can be regarded as not a force but a consequence of a curved space-time geometry where the source of curvature is the stress-energy tensor (representing matter, for instance). Thus, for example, the path of a planet orbiting around a star is the projection of a geodesic of the curved 4-D space-time geometry around the star onto 3-D space.
\end{abstract}

keywords Einstein-Klein-Gordon equations, metric functions, geodesics

\footnotetext{
*a.mahmoodzadeh130@gmail.com

†B.Malakolkalami@uok.ac.ir
} 


\section{Introduction}

As we know the most accepted cosmological models for understanding the mechanism of structure formation are those that contain dark matter and dark energy, with a possibly negative equation of state as the main constituents of the Universe. Both dark matter and dark energy can be described by a dynamical scalar field $\Phi$ that rolls in its potential $V(\Phi)[1,2]$. On the other hand, nowadays oscillatons are well known as astronomical objects with a spherically symmetric and time-dependent metrics which are asymptotically flat and non singular solutions to the Einstein-Klein-Gordon (EKG) equations and their properties have been the subject of recent studies as a possibility for the role of dark matter [3-6]. From astrophysical point of view oscillatons are gravitationally bound objects with the real scalar field $\Phi$ endowed with a scalar field potential $V(\Phi)$ which can be the subject of scalar field dark matter(SFDM) hypothesis at the galactic scale [7-9]. First of all we know that the space-time around an oscillaton varies with time depending on the form of the scalar field and scalar field potential. But on the other side we know that there is not yet a general agreement on the correct form that this scalar potential $V(\Phi)$ should have, see for instance [1] and references there in. In some previous works, for instance[10], has been suggested an exponential-like scalar field potential which fits very well the constraints from big bang nucleosynthesis, due to its (so called) tracker solutions, and then fine tuning can be avoided. The most simple example of a potential with both exponential behavior and a minimum is a cosh potential[11]. Recently we propose another form of the scalar field potential, described by $V(\Phi)=V_{0} e^{\lambda \sqrt{k_{0}} \Phi},[12]$ where $V_{0}$ and $\lambda$ are free parameters which should be fixed through the cosmological observation [13] or constraints which are imposed through the formulation of the problem[12]. Thus the geodesic motion of real particles around an oscillaton varies depending on the form of the potential. In previous works, the geodesics around an oscillaton made out of a quadratic $V(\Phi)=\frac{1}{2} m_{\Phi}^{2} \Phi^{2}$ scalar field potential has been carried out[14] and in this paper we are interested in to do this for the new exponential form of the potential mentioned above. Second if we assume that a galaxy is basically made out of scalar field dark matter described by an oscillaton, one would hope to observe periodic variations of stars' path around the galaxy. These two topics are the main motivations for studying what we are interested in. A summary of this study is as follows:

In section two we review the mathematical background of oscillatons endowed with an exponential scalar field potential $V(\Phi)$. In third section we solve the geodesic equations numerically and investigate their properties. In the last

part we summarize the results and make some final comments for next investigations.

\section{Mathematical background of oscillatons}

Oscillatons are time dependent spherically symmetric and asymptotically flat 
solutions for the coupled Einstein-Klein-Gordon (EKG) equations. Numerical solutions have been found for these equations by using the Fourier expansions. But non-linearity of these equations has caused only few modes have been employed $[13,16]$. The most general spherically symmetric metric is written as

$$
d s^{2}=g_{\alpha \beta} d x^{\alpha} d x^{\beta}=-e^{\nu-\mu} d t^{2}+e^{\nu+\mu} d r^{2}+r^{2}\left(d \theta^{2}+\sin ^{2} \theta d \varphi^{2}\right),
$$

where $\nu=\nu(t, r)$ and $\mu=\mu(t, r)$ are functions of time and radial position (the units are so chosen in which $\hbar=c=1$ ). The energy-momentum tensor endowed for a real scalar field $\Phi(t, r)$ with a scalar field potential $\mathrm{V}(\Phi)$ is defined as $[9]$

$$
T_{\alpha \beta}=\Phi_{, \alpha} \Phi_{, \beta}-\frac{1}{2} g_{\alpha \beta}\left[\Phi^{, \gamma} \Phi_{, \gamma}+2 V(\Phi)\right]
$$

The non-vanishing components of $T_{\alpha \beta}$ are

$$
\begin{aligned}
-T_{0}^{0} & =\rho_{\Phi}=\frac{1}{2}\left[e^{-(\nu-\mu)} \dot{\Phi^{2}}+e^{-(\nu+\mu)} \Phi^{\prime 2}+2 V(\Phi)\right], \\
T_{01} & =p_{\Phi}=\dot{\Phi} \Phi^{\prime} \\
T_{1}^{1} & =p_{r}=\frac{1}{2}\left[e^{-(\nu-\mu)} \dot{\Phi^{2}}+e^{-(\nu+\mu)} \Phi^{\prime 2}-2 V(\Phi)\right], \\
T_{2}^{2} & =p_{\perp}=\frac{1}{2}\left[e^{-(\nu-\mu)} \dot{\Phi^{2}}-e^{-(\nu+\mu)} \Phi^{\prime 2}-2 V(\Phi)\right],
\end{aligned}
$$

and we have also $T_{3}^{3}=T_{2}^{2}$. Over dots denote $\frac{\partial}{\partial t}$ and primes denote $\frac{\partial}{\partial r}$. The different components of the tensor for the scalar field mentioned above are identified as the energy density, the momentum density, the radial pressure and the angular pressure respectively. Einstein equations, $G_{\alpha \beta}=R_{\alpha \beta}-$ $\frac{1}{2} g_{\alpha \beta} R=k_{0} T_{\alpha \beta}$ are used to obtain differential equations for functions $\nu, \mu$, then

$$
\begin{aligned}
& (\nu+\mu)=k_{0} r \dot{\Phi} \Phi^{\prime}, \\
& \nu^{\prime}=\frac{k_{0} r}{2}\left(e^{2 \mu} \dot{\Phi}^{2}+\Phi^{\prime 2}\right), \\
& \mu^{\prime}=\frac{1}{r}\left[1+e^{\nu+\mu}\left(k_{0} r^{2} V(\Phi)-1\right)\right],
\end{aligned}
$$

where $R_{\alpha \beta}$ and $R$ are the Ricci tensor and Ricci scalar respectively and $k_{0}=8 \pi G=\frac{8 \pi}{m_{p l}^{2}}$, where the gravitational constant, $G$, is the inverse of the reduced Planck mass, $m_{p l}$, squared. The conservation of the scalar field tensor reads as

$$
T_{; \beta}^{\alpha \beta}=\Phi^{, \alpha}\left[\square \Phi-\frac{d V(\Phi)}{d \Phi}\right]=0
$$

where $\square=\partial_{\alpha} \partial^{\alpha}=g_{\alpha \beta} \partial^{\alpha} \partial^{\beta}(\alpha, \beta=0,1,2,3)$ is the d'Alembertian operator. Therefore we can obtain the Klein-Gordon (KG) equation for the scalar field $\Phi(t, r)$, 


$$
\Phi^{\prime \prime}+\Phi^{\prime}\left(\frac{2}{r}-\mu^{\prime}\right)-e^{\nu+\mu} \frac{d V(\Phi)}{d \Phi}=e^{2 \mu}(\ddot{\Phi}+\dot{\mu} \dot{\Phi}) .
$$

If we choose $\Phi(r, t)=\sigma(r) \phi(t)$, then Eq. (6) reads as

$$
\phi\left\{\sigma^{\prime \prime}+\sigma^{\prime}\left(\frac{2}{r}-\mu^{\prime}\right)\right\}-\lambda \sqrt{k_{0}} V_{0} e^{\nu_{0}+\mu_{0}} e^{\lambda \sqrt{k_{0}} \sigma(r) \phi(t)}=e^{2 \mu} \sigma(\ddot{\phi}+\dot{\mu} \dot{\phi}) .
$$

The following Fourier expansions

$$
e^{ \pm f(x) \cos (2 \theta)}=I_{0}(f(x))+2 \sum_{n=1}^{\infty}( \pm 1)^{n} I_{n}(f(x)) \cos (2 n \theta)
$$

where $I_{n}(z)$ are the modified Bessel functions of the first kind, require that Eq. (7) changes into

$$
\begin{aligned}
& \frac{1}{\sigma}\left\{\sigma^{\prime \prime}+\sigma^{\prime}\left(\frac{2}{r}-\mu^{\prime}\right)-\lambda \sqrt{k_{0}} V_{0} e^{\nu+\mu}\left(I_{0}\left(\lambda \sqrt{k_{0}} \sigma\right)+2 I_{1}\left(\lambda \sqrt{k_{0}} \sigma\right)\right)\right\}= \\
& \frac{e^{2 \mu}}{\phi}(\ddot{\phi}+\dot{\mu} \dot{\phi})+\frac{\lambda \sqrt{k_{0}} V_{0} e^{\nu+\mu}}{\sigma \phi}\left[I_{0}\left(\lambda \sqrt{k_{0}} \sigma\right)+2 I_{1}\left(\lambda \sqrt{k_{0}} \sigma\right)\right]
\end{aligned}
$$

It is necessary to recall that, we have taken into account only the first term of the exponential field expansion for simplicity to obtain Eq. (9). If we choose

$$
\sqrt{k_{0}} \Phi(r, t)=2 \sigma(r) \cos (\omega t)
$$

where $\omega$ is the fundamental frequency of the scalar field, then integrating on the Eq. (4.a) formally yields

$$
\nu+\mu=(\nu+\mu)_{0}+r \sigma \sigma^{\prime} \cos (2 \omega t)
$$

where $(\nu+\mu)_{0}$ is a function of r-coordinate only. The boundary conditions which will be discussed latter require that

$$
\nu_{1}+\mu_{1}=r \sigma \sigma^{\prime}
$$

It is useful to perform the following variable changes for numerical purposes of the following form

$$
x=m_{\Phi} r, \quad \Omega=\frac{\omega}{m_{\Phi}}, \quad e^{\nu_{0}} \rightarrow \Omega e^{\nu_{0}}, \quad e^{\mu_{0}} \rightarrow \Omega^{-1} e^{\mu_{0}} .
$$

Hence by using Eqs. (4.a-6), (10, 12) and setting each Fourier component to zero the metric functions and the radial part of the scalar field are obtained as

$$
\begin{aligned}
& \nu_{0}^{\prime}=x\left[e^{2 \mu_{0}} \sigma^{2}\left(I_{0}\left(2 \mu_{1}\right)-I_{1}\left(2 \mu_{1}\right)\right)+\sigma^{\prime 2}\right], \\
& \nu_{1}^{\prime}=x\left[e^{2 \mu_{0}} \sigma^{2}\left(2 I_{1}\left(2 \mu_{1}\right)-I_{0}\left(2 \mu_{1}\right)-I_{2}\left(2 \mu_{1}\right)\right)+\sigma^{\prime}\right], \\
& \mu_{0}^{\prime}=\frac{1}{x}\left\{1+e^{\nu_{0}+\mu_{0}}\left[x^{2}\left(I_{0}\left(x \sigma \sigma^{\prime}\right) I_{0}(2 \lambda \sigma)+2 I_{1}\left(x \sigma \sigma^{\prime}\right) I_{2}(2 \lambda \sigma)\right)-I_{0}\left(x \sigma \sigma^{\prime}\right)\right]\right\},
\end{aligned}
$$


$\mu_{1}^{\prime}=\frac{2}{x} e^{\nu_{0}+\mu_{0}}\left[x^{2}\left(I_{0}\left(x \sigma \sigma^{\prime}\right) I_{2}(2 \lambda \sigma)+I_{1}\left(x \sigma \sigma^{\prime}\right) I_{0}(2 \lambda \sigma)+I_{2}\left(X \sigma \sigma^{\prime}\right) I(2 \lambda \sigma)\right)-\right.$
$\left.I_{1}\left(x \sigma \sigma^{\prime}\right)\right]$,

$\sigma^{\prime \prime}=-\sigma^{\prime}\left(\frac{2}{x}-\mu_{0}^{\prime}-\frac{1}{2} \mu_{1}^{\prime}\right)+\lambda e^{\nu_{0}+\mu_{0}} I_{0}(2 \lambda \sigma)\left(I_{0}\left(x \sigma \sigma^{\prime}\right)+I_{1}\left(x \sigma \sigma^{\prime}\right)\right)-$ $e^{2 \mu_{0}} \sigma\left[I_{0}\left(2 \mu_{1}\right)\left(1-\mu_{1}\right)+I_{1}\left(2 \mu_{1}\right)+I_{2}\left(2 \mu_{1}\right)\right]$.

Now primes denote $\frac{d}{d x}$. The two last equations are true if we put, $k_{0} V_{0}=$ $m_{\Phi}^{2}$. With initial boundary values for $\nu_{0}(x=0), \nu_{1}(x=0), \mu_{0}(x=0)$, $\mu_{1}(x=0), \sigma(x=0)$ and parameter $\lambda$, these equations are soluble numerically and the answers are in the form of Interpolation Functions. Therefore the metric functions and then the metric coefficients are completely determined. Singularity points for the metric functions are also obtained through boundary conditions. For example if we put $\nu_{0}(x=0)=-0.11, \nu_{1}(x=0)=0.17$, $\mu_{0}(x=0)=0.11, \mu_{1}(x=0)=-0.17, \sigma(x=0)=0.3$, and $\lambda=-0.1$ then singularity happens at point $x=1.62380$. ( with increasing the values of $\lambda$ and $\sigma$, singularity points will happen at less and less values of $x$ ). The important point that should be mentioned here is that the exponential and quadratic scalar potentials described by $V(\Phi)=V_{0} e^{\lambda \sqrt{k_{0}} \Phi}$ and $V(\Phi)=\frac{1}{2} m_{\Phi}^{2} \Phi^{2}$ respectively with the same initial boundary conditions cause different metric functions and metric coefficients $[4,12,16]$.

For next purposes the answers obtained from Eqs. (14-17) are fitted to six order which are in compliance with equations (19-22). The results of these fittings are shown in Fig. 1. From now on the metric functions are presented by these fitted functions of the following form:

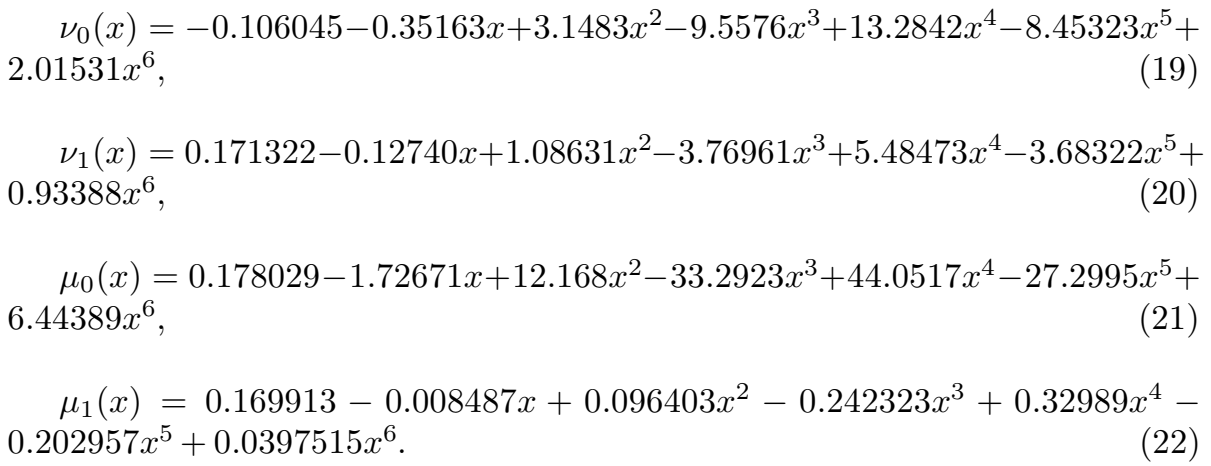



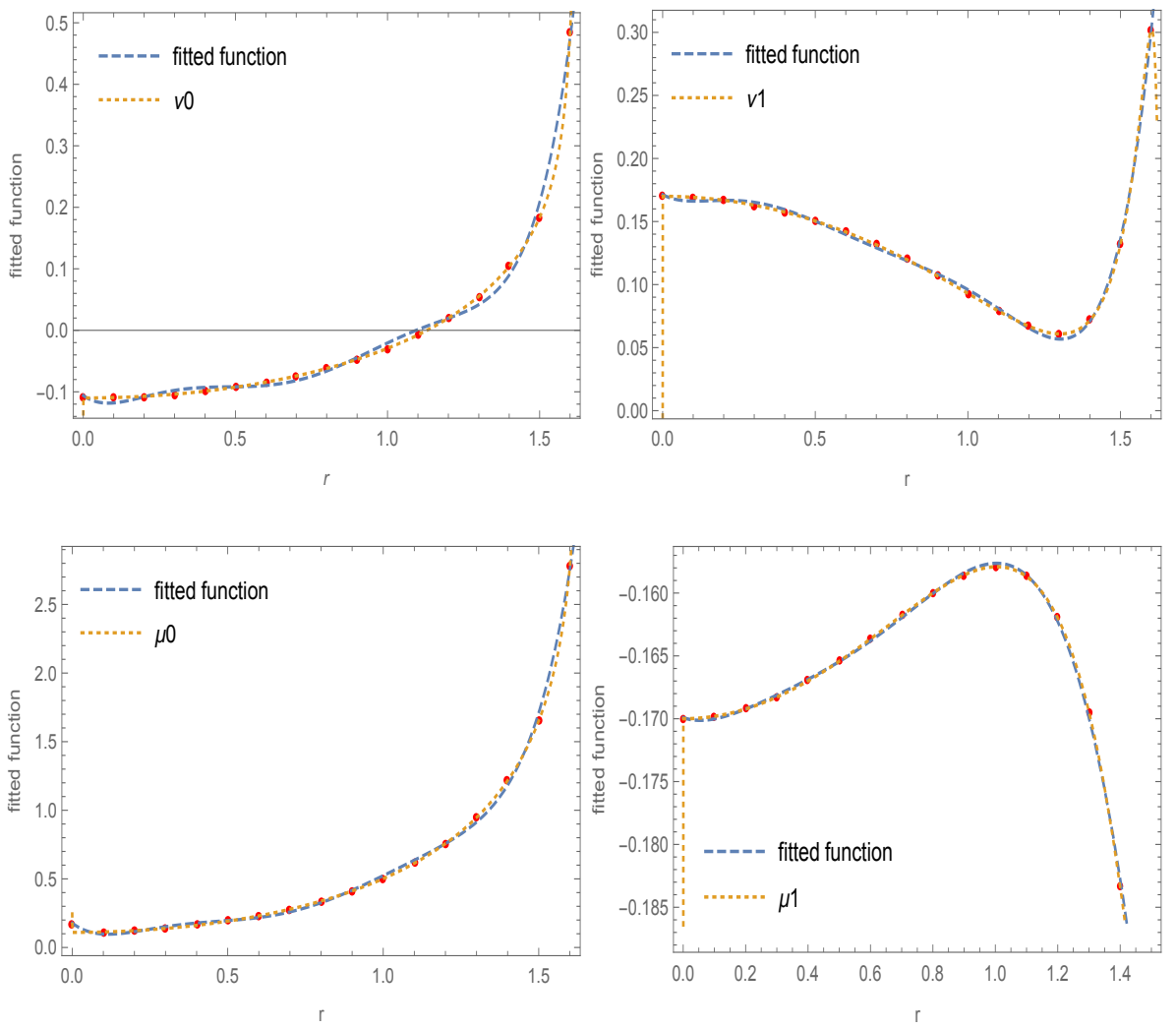

Fig. 1. The metric functions and their corresponding fitted functions.

The next step is to specify the geodesics around oscillatons for real particles. As we mentioned above, this work for a real particles around oscillatons described by a quadratic scalar field potential has been done in previous work[14] and investigation of geodesics for real particles around oscillatons made of an exponential scalar field potential discribed by[12], is the subject of this paper. For this work we continue the process as follow:

If we redefine the metric coefficients as the following form

$$
g_{t t}=-e^{\nu-\mu}=-B(x, t), \quad g_{r r}=e^{\nu+\mu}=A(x, t), \quad C(x, t)=\frac{A(x, t)}{B(x, t)},
$$

then the Einstein equations can be rewritten as

$$
\begin{aligned}
& \frac{\partial A}{\partial t}=k_{0} A \frac{\partial \Phi}{\partial t} \frac{\partial \Phi}{\partial x}, \\
& \frac{\partial A}{\partial x}=\frac{k_{0} A x}{2}\left[C\left(\frac{\partial \Phi}{\partial t}\right)^{2}+\left(\frac{\partial \Phi}{\partial x}\right)^{2}+2 A V(\Phi)\right], \\
& \frac{\partial C}{\partial x}=\frac{2 C}{x}\left[1+A\left(k_{0} x^{2} V(\Phi)-1\right],\right.
\end{aligned}
$$




$$
C \frac{\partial^{2} \Phi}{\partial t^{2}}=-\frac{1}{2} \frac{\partial C}{\partial t} \frac{\partial \Phi}{\partial t}+\frac{\partial^{2} \Phi}{\partial x^{2}}+\frac{\partial \Phi}{\partial x}\left[\frac{2}{x}-\frac{1}{2 C} \frac{\partial C}{\partial x}\right]-A \frac{d V(\Phi)}{d \Phi} .
$$

It is clear that by combining of Eq. (24. c) and (24. d) the non-linearity of these equations can be minimized. By applying $A(x, t), B(x, t)$ and $C(x, t)$ the non-vanishing components of $T_{\alpha \beta}$ can be renovated as

$$
\begin{aligned}
& -T_{0}^{0}=\rho_{\Phi}=\frac{1}{2}\left[\frac{C(x, t)}{A(x, t)}\left(\frac{\partial \Phi}{\partial t}\right)^{2}+\frac{1}{A(x, t)}\left(\frac{\partial \Phi}{\partial x}\right)^{2}+2 V(\Phi)\right], \\
& T_{1}^{0}=p_{\Phi}=\left(\frac{\partial \Phi}{\partial t}\right)\left(\frac{\partial \Phi}{\partial x}\right), \\
& T_{1}^{1}=p_{r}=\frac{1}{2}\left[\frac{C(x, t)}{A(x, t)}\left(\frac{\partial \Phi}{\partial t}\right)^{2}+\frac{1}{A(x, t)}\left(\frac{\partial \Phi}{\partial x}\right)^{2}-2 V(\Phi)\right], \\
& T_{2}^{2}=p_{\perp}=\frac{1}{2}\left[\frac{C(x, t)}{A(x, t)}\left(\frac{\partial \Phi}{\partial t}\right)^{2}-\frac{1}{A(x, t)}\left(\frac{\partial \Phi}{\partial x}\right)^{2}-2 V(\Phi)\right] .
\end{aligned}
$$

\subsection{Boundary Conditions And Numerical Results}

We can solve the (EKG) equations by considering the following Fourier expansions [13].

$$
\begin{aligned}
& \sqrt{k_{0}} \Phi(x, t)=2 \sigma(x) \cos (\omega t), \\
& A(x, t)=\sum_{n=0}^{n_{\text {max }}} A_{n}(x) \cos (n \omega t)=e^{\nu+\mu}= \\
& e^{\nu_{0}+\mu_{0}}\left[I_{0}\left(\nu_{1}+\mu_{1}\right)+2 \sum_{n=1}^{n_{\max }} I_{n}\left(\nu_{1}+\mu_{1}\right) \cos (2 n \omega t)\right], \\
& C(x, t)=\sum_{n=0}^{n_{\max }} C_{n}(x) \cos (n \omega t)=e^{2 \mu}= \\
& e^{2 \mu_{0}}\left[I_{0}\left(2 \mu_{1}\right)+2 \sum_{n=1}^{n_{\text {max }}} I_{n}\left(2 \mu_{1}\right) \cos (2 n \omega t)\right],
\end{aligned}
$$

where $n_{\max }$ is the Fourier mode at which the series are truncated. It is remarkable that in Eq. (26 a) we have used the first term of the scalar field ,$\sqrt{k_{0}} \Phi(x, t)=\sum_{n=1}^{n_{\max }} \sigma(x) \cos (n \omega t)$, for simplicity, because the first term of the approximation is sufficient to yield the main properties of oscillaton[16]. Nonsingularity and asymptotically flatness are two main characteristics of an oscillaton which determine the boundary conditions. Solutions of (EKG) equations must be regular at $x=0$, this means that $\sigma^{\prime}(0)=0$ and $A(x=0)=0$. The latter condition is equivalent to $\nu_{0}(0)+\mu_{0}(0)=0, \nu_{1}(0)+\mu_{1}(0)=0$ and $A_{n>0}(x=0)=0$. The conditions $\sigma(\infty)=0$ and $A(x=\infty, t)=1$ are imposed by asymptotically flatness solutions as well as $A_{0}(\infty)=1, A_{n>0}(\infty)=0$, these conditions are equivalent to $\nu_{0}(\infty)+\mu_{0}(\infty)=0, \nu_{1}(\infty)=0$ and $\mu_{1}(\infty)=0$. But the condition of $\nu_{0}(\infty)+\mu_{0}(\infty)=0$ yields $C_{n>0}(\infty)=0$ and $C_{0}(\infty) \neq 1$ because 
this variable determines the fundamental frequency $\Omega=\sqrt{C_{0}(\infty)}=e^{-\nu_{0}(\infty)}$ as an output value after solving the oscillaton equations. The next step is to choose $\sigma(0)$ as the central value and $C_{n \geq 2}$ for fulfilling of boundary conditions, Thereby we obtain a set of eigenvalues for each central value. It is quotable that Eqs. (26. b) and (26. c) confine the metric coefficients to even nodes only.

\section{Geodesic equations of motion}

Spherically symmetric metric allows us to write the geodesics equations for the metric (1). By using the geodesics equations which are in the form of

$$
\frac{d^{2} x^{\kappa}}{d \tau^{2}}=-\Gamma_{\mu \nu}^{\kappa} \frac{d x^{\mu}}{d \tau} \frac{d x^{\nu}}{d \tau},
$$

where $\Gamma_{\mu \nu}^{k}=\frac{1}{2} g^{k \eta}\left(-g_{\mu \nu, \eta}+g_{\nu \eta, \mu}+g_{\eta \mu, \nu}\right)$ is affine connection, $\kappa, \eta, \mu, \nu=0$, $1,2,3$ and $x^{\kappa}, x^{\mu}, x^{\nu}=(t, r, \theta, \phi)$, for a special case $\left(\theta=\frac{\pi}{2}\right)$ we have:

$$
\begin{aligned}
& \ddot{t}=-\frac{1}{2 B} \frac{\partial B}{\partial t} \dot{t}^{2}-\frac{1}{B} \frac{\partial B}{\partial r} \dot{r} \dot{t}-\frac{1}{2 B} \frac{\partial A}{\partial t} \dot{r}^{2}, \\
& \ddot{r}=-\frac{1}{2 A} \frac{\partial B}{\partial r} \dot{t}^{2}-\frac{1}{A} \frac{\partial A}{\partial t} \dot{r} \dot{t}-\frac{1}{2 A} \frac{\partial A}{\partial r} \dot{r}^{2}+\frac{r}{A} \dot{\phi}^{2}, \\
& \ddot{\phi}=-\frac{2}{r} \dot{r} \dot{\phi},
\end{aligned}
$$

where dot is derivative with respect to proper $\operatorname{time}(\tau)$.

These equations are soluble numerically because it is not possible to write the radial equation (28 b) as usual as $\frac{1}{2} \dot{r}^{2}+U(r)=E$ where $\frac{1}{2} \dot{r}^{2}$ is the kinetic energy, $U(r)$ an effective potential and $E$ the particles total energy. The condition $L=0$ equivalent to $\phi=\phi_{0}=0$ means that a particle undergoes a direct straight line free fall from its initial position $\left(r_{0}, \phi_{0}\right)$. For $L \neq 0$ a particle will undergo a variety of paths, on the other hand for $L>0$ with increasing $\phi$, particles undergo in the counter clock wise direction but for $L<0$ and with increasing $\phi$ , particles undergo in the clock wise direction. Needless to say that the motion depends on the central value of the radial part of the scalar field, $\sigma(x=0)$, the angular momentum $L$, the initial radial position $r_{0}$ and initial radial speed $\dot{r}_{0}$. In turning point where $\dot{r}_{0}=0$, one of these two options happen: either a particle goes away to infinity or oscillates with a certain period and amplitude which could be found by solving the geodesic equations numerically. Fig. 2 . shows a path at which a particle goes away to infinity gradually under the initial conditions $r_{0}(\tau=0)=1.63, \dot{r}_{0}(\tau=0)=0$ and two different values for momentum , $L=1.5$ and 2.8 for the case $\sigma(x=0)=0.3$ (at which singularity happens at point $x=1.62380$ ) for a range of proper time $[\tau=0,40]$. As we can see with increasing $\tau$, the path of the particles approaches to a closed circles. Changing the radial position to $r_{0}(\tau=0)=2.63$ and momentum $L=1.5$ and $L=2.8$ but this time the proper time $\tau=100$ causes particles undergo closed orbital. See Fig. 3. 

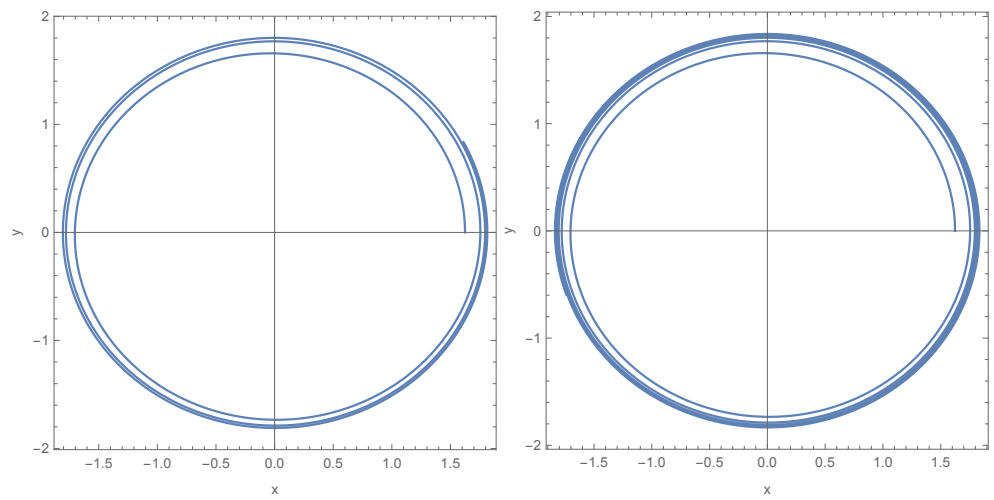

Figs. 2. The orbits for particles under the boundary conditions: $\sigma(x=0)=$ $0.3, r_{0}(\tau=0)=1.62381, \dot{r}_{0}(\tau=0)=0$ and $L=1.5$ for left plot and $L=2.8$ for right plot at which proper time varies from zero to 40 .
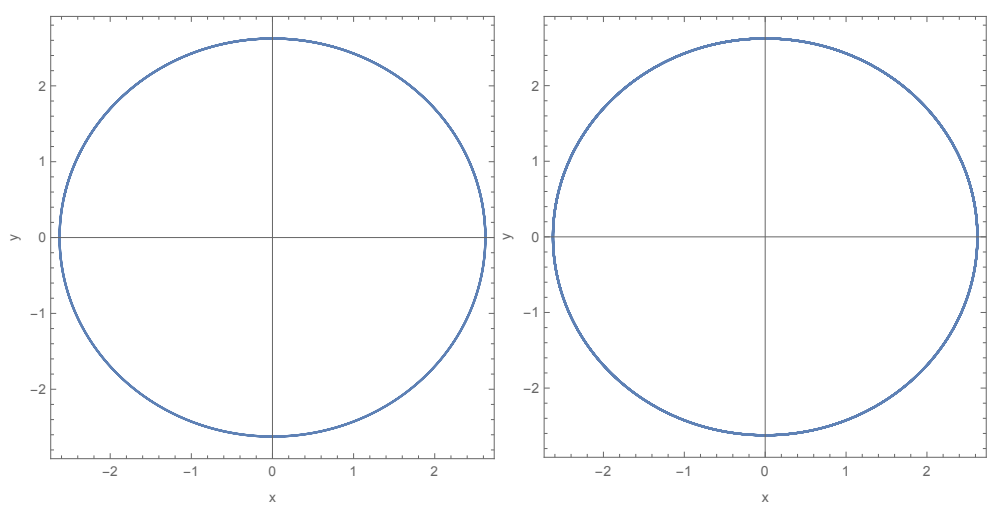

Fig. 3. The orbits for particles under the boundary conditions: $\sigma(x=0)=$ $0.3, r_{0}(\tau=0)=2.62381, \dot{r}_{0}(\tau=0)=0$ and $L=1.5$ for left plot and $L=2.8$ for right plot at which proper time varies from zero to 100 .

Reiteration of the results mentioned above but this time for $\sigma(x=0)=0.7$ and the same $\lambda=-0.1$ show that singularity happens at point $x=1.43696$ for metric functions, therefore for initial values of $r_{0}(\tau=0)=1.44, \dot{r}_{0}(\tau=0)=0$ and two different values for momentum , $L=1.5$ and $L=2.8$,when proper time varies from zero to 40, the geodesics for real particles are shown in Fig. 4 and Fig. 5. 

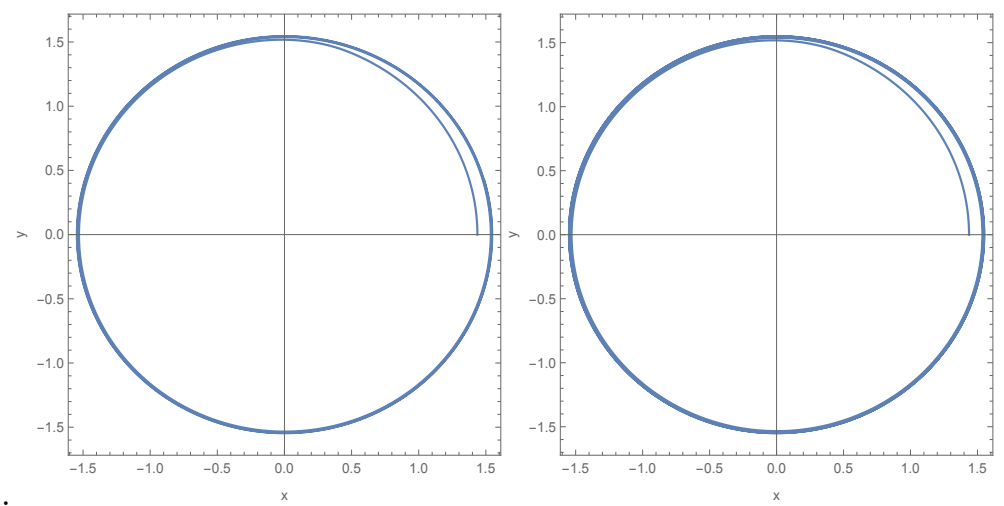

Fig. 4. The orbits for particles under the boundary conditions: $\sigma(x=0)=$ $0.7, r_{0}(\tau=0)=1.43697, \dot{r}_{0}(\tau=0)=0$ and $L=1.5$ for left plot and $L=2.8$ for right plot at which proper time varies from zero to 40 .
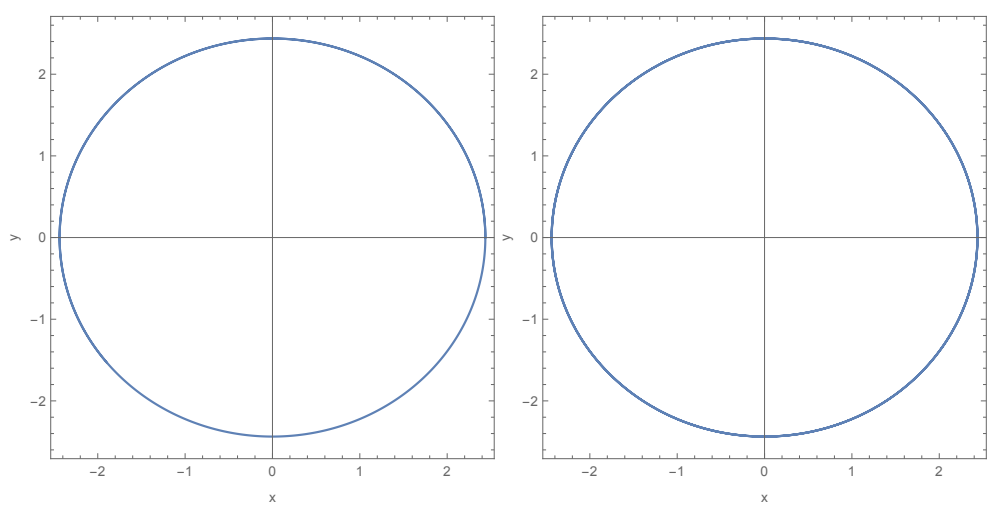

Fig. 5. The orbits for particles under the boundary conditions: $\sigma(x=0)=$ $0.7, r_{0}(\tau=0)=2.43697, \dot{r}_{0}(\tau=0)=0$ and $L=1.5$ for left plot and $L=2.8$ for right plot at which proper time varies from zero to 40 .

\section{4. conclusions}

In this study we presented the simplest approximation for solving a minimal coupled Einstein-Klein-Gordon equations for a spherically symmetric oscillating soliton object called oscillaton endowed with an exponential scalar field potential $V(\Phi)=V_{0} e^{\lambda k_{0} \Phi}$ and an harmonic time-dependent scalar field $\Phi(r, t)=2 \sigma(r) \cos (\omega t)$. By taking into account the Fourier expansions of differential equations and with regard to the boundary conditions which require the non singularity and asymptotically flatness, the solutions ( the metric functions) are obtained numerically. It is more convenient to fit these solutions which are in the form of Interpolation Functions to new functions, In present study this work has been done to order four, although better results are obtained by taking 
into account the higher order of fitting. By determination of the metric functions, the metric coefficients are also obtained easily. As we know the metric coefficients are the main tools for dealing with the geodesic equations according

to $\frac{d^{2} x^{k}}{d \tau^{2}}+\Gamma_{\mu \nu}^{k} \frac{d x_{\mu}}{d \tau} \frac{d x_{\nu}}{d \tau}=0$. Solving these equations based on initial boundary conditions and plot the answers in the polar coordinates show the trajectory of the real particles. This work has been done at points very close to what is called the singularity for one of the metric functions for two different central values of $\sigma(x=0)=0.3$ and 0.7 and momentum values $L=1.5$ and 2.8 at turning points where $\dot{r}_{0}=0$, the particle's speed, is equal to zero. Results show that for radial points outside the oscillaton and close to the singularity point the particles' trajectory gradually increase or decrease for positive and negative values of the momentum respectively and for points sufficiently far from the oscillton the particles'path are closed orbits. Despite the presented results in this study, there are some problems that should be investigated elsewhere in the future works. 1. Under which conditions and for which values of $\sigma, L$, and other initial values the radial coordinates of particles oscillate in time. 2 . If the radial coordinates of the particles oscillate in time then the amplitude of oscillation and the period of oscillations are desirable.

\section{References}

[1] Abril Suárez, Introduction to Scalar Field Dark Matter Structure Formation, Journal de Ciencia e Ingeniería, Vol.4, No.1, August 2012, pp. 1 - 8 Investigación.

[2] Juan Magaña, Tonatiuh Matos, Abriz Suárez, F. J. Sánchez-Salcdo, arXiv: 1204.5255v2[astro.-ph. Co](2012).

[3] E. Saidel and W-M.Suen, Phys. Rev. Lett. 66.1659 (1991). E. Saidel and W-M.Suen, Phys. Rev. Lett. 72. 2516(1994).

[4] L. A. Ureña-López Class. Quantum Grav. 19 , 2617 (2002).

[5] M. Alcubierre , F. S. Guzmán , L. A. Ureña-López Class. Quantum Grav. 19, 5017 (2002). F. S. Guzmán, L. A. Ureña-López (2004) Phys. Rev. D 69, 124033.

[6] Tonatiuh Matos, Alberto Vázquez-Gonzá lez and Juan Magaña, Mon. Not. R. Astron. Soc. 393, 1359-1369 (2009).

[7] T. Matos and F. S. Guzmán Class. Quantum Grav. 18. 5055 (2001).

[8] T. Matos, F. S. Guzmán, L. A. Ureña-López and D. Núñez pre prent astro-ph/0102419.

[9] T. Matos, M. Alcubierre and D. Núñez. To be published. M. Alcubierre, T. Matos, D. Nunez , L. A. Ureña-López and P. wieder hold, preprint gr-qc/0110102. 
[10] Tonatiuh Matos, Luis Arturo Ureña-Lõpez and et al.,The Scalar Field Dark Matter Model: A Braneworld Connection, Lect. Notes Phys. 646, 401-420 (2004), Springer-Verlag Berlin Heidelberg, 2004.

[11] Tonatiuh Matos and F. Siddhartha Guzmán, arXiv: gr-qc/0108027v1, 2001.

[12] B. Malakolkalami, A. Mahmoodzadeh. Phys. Rev. D. 94.103505, 2016.

[13] T. Matos and L. A. Ureña-López, Phys. Rev. D 63, 63506 , 2001.

[14] R. Becerril, T. Matos, L. A. Ureña-López, Gen. Relative. Gravit. (2006) 38 (4). 633-641.

[15] L. A. Ureña-López, T. Matos, R. Becerril. Class. Quantum Grav. 19. 62596277, 2002.

[16] L. A. Ureña-López gr-qc/0104093v3, 2002. 